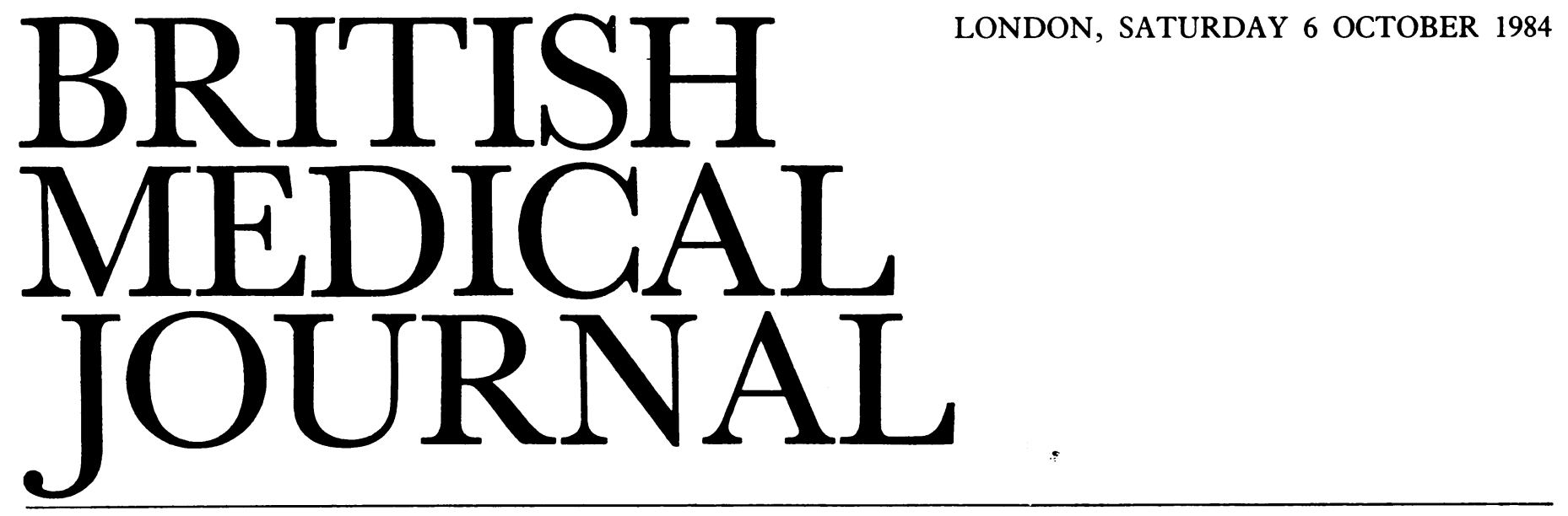

\title{
Failures of the cervical cytology screening programme
}

To suppose that any non-communicable disease in adults could be completely controlled by a screening programme is wishful thinking. Failures of the system are inevitable, arising from failure to reach all of the target population, failure of the screening test's sensitivity and frequency to detect all the cases, failure to implement treatment in all cases detected, and failure of treatment to modify the natural course of the disease. The cervical cytology screening programme in Britain, which is demonstrably less effective than in some other countries, ${ }^{1}$ suffers from all these deficiencies. Two papers in this issue report studies describing some of these defects, a third proposes a system for a more efficient screening service, and a fourth considers the problem in general practice.

Elwood et al (p 891) have assessed the adequacy of management of over 1000 women who had a first abnormal smear result in Nottingham in 1981, finding that fewer than two thirds had received the recommended follow up. This failure occurred despite the presence of a well organised centralised cytology laboratory with an explicit follow up policy for different levels of cytological abnormality. The findings are similar to those in a survey in north London, ${ }^{2}$ with deficiencies in communication among different sectors of the health service, and between doctor and patient accounting for a much higher proportion of failed follow ups than patient default. Nevertheless, the last group contains a recognised high risk group for cervical cancer-namely, women attending special clinics. The need for maintaining confidentiality of such attendances may not be in the patient's best interests so far as prevention of invasive cervical carcinoma is concerned. By implication most of the patients for whom appropriate action had not been taken had milder degrees of cellular abnormality (dyskaryosis), in which the recommendation was for repeat smears, two consecutive negative reports being required before discharging a patient from follow up. Though these women may not be in imminent danger, the authors rightly draw attention to the risk of invasive carcinoma of the cervix in patients with untreated intraepithelial neoplasia. ${ }^{3}$ Their proposed solution to achieve more comprehensive follow up is, firstly, for a computerised record system providing a cumulative record of all successive tests for each woman, preferably linked to her family practitioner committee record so that changes of name, of general practitioner, and of place of residence may be determined; and, secondly, building in "fail safe" signals to alert the laboratory when there is evidence that the recommended management has not been implemented by the proposed time.

Paterson et al (p 896) address the question of the sensitivity of the cervical smear test and the frequency with which screening should be done. They tried to examine the screening history of 500 women in Yorkshire who had been diagnosed as having invasive carcinoma of the cervix between 1968 and 1980, although, in the event, they could obtain information on only 312 of them. If we assume that the distribution of smears in the untraced sample was similar to that where information was available $19 \%$ of this series of patients had had a negative result of a cervical smear test within five years of the diagnosis of invasive cancer-that is, within the rescreening interval currently recommended by the Department of Health and Social Security. ${ }^{4}$ These "false negative" results, however, need to be assessed in the context of all women from the same population in the same time period who were tested and found to have intraepithelial neoplasia ("true positives") before any assessment of the sensitivity of the test is possible; unfortunately, this information is not available. Other aspects of this study also suffer from a lack of knowledge of denominators, even the comparisons of trends in age specific incidence and mortality being based on numbers and not rates.

Some have suggested that false negative cervical cytology reports are more likely to be due to an error in taking the smear than to a laboratory error. ${ }^{5}$ Nevertheless, 58 negative smears from these patients were subjected to independent review and, perhaps with the benefit of knowing the ultimate diagnosis, $34(59 \%)$ were deemed positive and a further 11 (19\%) unsatisfactory, leaving only $13(22 \%)$ as confirmed negatives. Surprisingly, there was no apparent trend indicating a higher proportion of positive verdicts closer to the time of diagnosis, although the number of slides available for review was smaller the longer the duration of time before diagnosis. The authors suggest that progression from a negative smear to invasive cancer occurs more rapidly in younger women than in older ones, although no data are presented to support this; the fact that more women under 35 had a negative smear almost certainly reflects the fact that more young women than old are screened. Moreover, there was a more favourable stage distribution-and fewer deaths- 
among women with one or more previous negative smears. Women who are already in the screening system are probably more likely to have an invasive cancer diagnosed at an earlier stage than those who are not.

A further conclusion which may be drawn from this paper is that some $80 \%$ of patients developing invasive cancer did not have a previous negative screening test result, and hence must have slipped through the screening system because of failure to be screened at all, failure of adequate follow up, or failure of treatment. One case in the last category is quoted where a woman had been treated for intraepithelial neoplasia by cone biopsy and, despite six subsequent negative smears, eventually developed invasive cancer.

Though it is important to maintain high quality control at every stage of screening, the priority to be given to different measures to improve quality needs to be assessed in the light of the contribution of each to controlling the disease. Two recent studies, in Manchester and in south London, have looked at the reasons why women developing invasive cancer of the cervix had not been detected at a preinvasive stage by screening. ${ }^{67}$ By far the most common reason was that they had never been screened at all; this applied to two thirds of the total sample and four fifths of those over 40, among whom the great majority of cases occur. Failure to follow up abnormal cytological results accounted for about $15 \%$, with too infrequent screening - that is, an interval of over five years-and possible false negative results each contributing less than $10 \%$ of the total.

Hence in deciding priorities, recruitment of women into the screening system, particularly those past their childbearing years, still offers the best prospect of preventing a substantial number of women from developing invasive cancer. This is not to deny the increase in the incidence of the disease in younger women or to suggest that they should not be screened at all: rather to emphasise that screening resources should be more equitably shared among all those at risk. This is the essential message of the third paper in this week's issue, a statement by the ICRF Coordinating Committee on Cervical Screening ( $p$ 894). This outlines a plan for positive invitations to all adult women to be screened every five years, using a computerised invitation and recall scheme integrated with laboratory records, and incorporating a system that monitors that action has been taken on positive results. Such a scheme has been developed by the Exeter Family Practitioner Services Computer Unit, funded by the Department of Health and Social Security, and is now starting to be implemented in a few family practitioner committee areas. It has not yet been tested in practice and it remains to be seen whether exerting more positive control of the screening programme by a computerised system will make a sizable impact on the incidence of cervical cancer.

Meanwhile, as the fourth paper in this issue ( $p$ 883) shows, general practitioners can persuade virtually all the women in their practices to have smears. The $96 \%$ uptake rate reported by Standing and Mercer is attributable to their determination - culminating in home visits for women who did not respond to letters and telephone calls.

The principal reason for choosing a five yearly rescreening interval is cost. The clinician, whose priority is to do the best he can for his individual patient, may prefer to reconimend a shorter rescreening interval, as suggested by Paterson et al, but even one yearly screening will miss some fast growing cancers and endocervical adenocarcinomas. Health authorities, however, have a responsibility to the whole population they serve, and from this public health point of view a five yearly programme which reaches a high proportion of women and ensures appropriate action on those found positive will $\underline{\underline{T}}$ clearly prevent many more cases of invasive cancer than $\vec{s}$ frequently repeated smears on a small number of women. $\stackrel{\circledR}{\unrhd}$ Authorities may look at the priority to be given to prevention of cervical cancer (2000 deaths a year in England and Wales) alongside that of other preventive programmes for women, $D$ including lung cancer ( 8400 female deaths) and accidents $\mathbb{\infty}$ (8500 female deaths). In this context the provision of a $\frac{\mathbb{Q}}{2}$ computerised system to implement and monitor five yearly screening of all adult women seems a very reasonable policy. $\overrightarrow{\vec{\omega}}$ Let us hope that it will result in a reduction in incidence of $\overrightarrow{0}$ invasive cervical cancer comparable with that achieved in other countries.

JOCELYN CHAMBERLAIN

Regional Specialist in Cancer Services,

South West Thames Regional Cancer Organisation,

Royal Marsden Hospital,

Sutton SM2 5PT

1 Hakama $M$. Trends in incidence of cervical cancer in the Nordic countries. In: Magnus K, ed. Trends in cancer incidence. Washington: Hemisphere, 1982:279-92.

Armstrong A Follow up of women with dysplasia of the uterine cervix. London: University of

London, 1980. (PhD thesis.)

Kinlen LJ, Spriggs AI. Women with positive cervical smears but without surgical intervention. A follow up study. Lancet 1978;ii:463-5.

Department of Health and Social Security Health Services Development. Screening for cervical cancer. London: DHSS, 1984. (HC84(17).

Yule R. The prevention of cancer of the cervix by cytological screening of the population. In Easson EC, ed. Cancer of the uterine cervix. London: Saunders, 1984:11-25.

6 Chisholm DK, Haran D. Cases of invasive cervical cancer in the north west in spite of screening. British fournal of Family Planning 1984;10:3-8.

Ellman R, Chamberlain J. Improving the effectiveness of cervical cancer screening. $7 \mathrm{R}$ Coll Gen Pract (in press).

\section{Children on motorcycles: a cause for concern}

Motorcycles are known to be dangerous machines, but in recent years much has been done to reduce the number of accidents, particularly among teenage riders, who account for almost half of all motorcycle casualties. ${ }^{1}$ Little or no attention has, however, been given to the injuries sustained by the ever growing numbers of children riding motorcycles for sport and pleasure. Sherman and Mackinnon's paper (p 877) presents some alarming data. Although their survey included only relatively few patients, it clearly showed the potential for serious injury for both the supervised and 3 unsupervised riders. They rightly emphasise the need for $ᄋ$ further study: their experiences are unlikely to be unique, and probably cases of the kind they describe are occurring throughout Britain.

By law children aged under 16 are forbidden to ride a $>$ powered two wheeled vehicle on the public highway, but that does not prevent their riding motorcycles on private pro- $N$ perty. Since the Autocycle Union established its youth division in 1974, clubs catering for motorcycle riders aged $\bar{\sigma}$ between 6 and 17 have proliferated. The rules governing the conduct of the members of these clubs are comprehensive and ensure that children ride with maximum safety. Many $\stackrel{+}{+}$ more children, however, ride unsupervised in fields and on waste and common ground. In these circumstances the pro- $\frac{\overrightarrow{\mathbb{D}}}{\mathrm{D}}$ vision of a safe motorcycle, adequate training, and protective $\frac{\vec{\Phi}}{\mathbb{D}}$ clothing becomes the sole responsibility of the parents or guardians.

We have few data on the injuries sustained during the $\frac{\delta}{0}$ pursuit of this activity; the details that are available relate only to motorcycle accidents occurring on the public highway, and even then non-fatal accidents are underreported. ${ }^{2}$ 